\title{
Local modification of magnetic properties by an electron beam
}

\author{
R. Allenspach, ${ }^{\text {a) }}$ A. Bischof, U. Dürig, and P. Grütter ${ }^{\text {b) }}$ \\ IBM Research Division, Zurich Research Laboratory, CH-8803 Rüschlikon, Switzerland
}

(Received 27 May 1998; accepted for publication 12 October 1998)

\begin{abstract}
Magnetic anisotropies can be modified locally by intense electron beams with keV energy. In ultrathin epitaxial Co films grown on $\mathrm{Pt}(111)$, switching of the easy axis from parallel to perpendicular to the surface can be induced, and the domain size can be enlarged by one order of magnitude. (C) 1998 American Institute of Physics. [S0003-6951(98)03150-7]
\end{abstract}

Control of magnetic anisotropy provides a means to investigate basic concepts in low-dimensional magnetic systems as well as a tool for tuning magnetic properties for possible applications. A striking effect associated with magnetic anisotropy is the one accompanied by a change of the easy magnetization direction. An increase of the perpendicular magnetic surface anisotropy can lead to a complete reorientation of the magnetization from parallel to perpendicular to a film surface. It can be induced by depositing metal layers ${ }^{1}$ or by annealing. ${ }^{2}$ Switching within the plane is also provoked by chemisorption of gases in the $\mathrm{Co} / \mathrm{Cu}$ system. ${ }^{3,4}$ Ion etching, on the other hand, has the opposite effect of reducing perpendicular anisotropy. ${ }^{5}$ These experiments have altered film properties on a macroscopic length scale. On a local scale, modifications of the magnetic properties have been induced by small amounts of metallic adsorbates ${ }^{6}$ and, very recently, selective ion bombardment has been successfully used for magnetic patterning of Co films. ${ }^{7}$

Here we present experiments which show that modifications on a local scale are possible without adding or removing atoms or molecules. To "write" the modified magnetic properties into an ultrathin Co film, we use the electron beam of a scanning electron microscope (SEM). In this way, the magnetization direction can be forced to change locally, and magnetic domain sizes can be expanded by up to one order of magnitude. Possible origins of this induced change of magnetic properties are discussed and tested by experiments.

The Co films were grown in situ by molecular beam epitaxy at a rate of $0.5-1 \mathrm{ML} / \mathrm{min}$ [1 ML (monolayer) $=0.205 \mathrm{~nm}$ ] on a Pt single-crystalline substrate kept at room temperature. Substrate preparation followed the recipes developed earlier. ${ }^{8}$ The Pt substrate is nominally oriented along the (111) direction. By selecting different areas on the substrate, a miscut from the (111) direction of between $0.5^{\circ}$ and $4^{\circ}$ can be chosen. These areas are identified in scanning tunneling microscopy experiments by a varying density of steps of monoatomic height. The data presented all refer to a Co film thickness of 3.8 ML. At this thickness the magnetization direction can be at any angle, from parallel to perpendicular to the surface, depending on the local step density. ${ }^{9}$

All local modifications leading to changes of magnetic properties were generated by the electron beam of our spin-

\footnotetext{
${ }^{a)}$ Electronic mail: ral@zurich.ibm.com

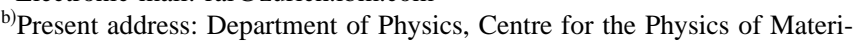
als, McGill University, Montréal H3A 2T8, Canada.
}

polarized SEM (spin-SEM) ${ }^{10}$ with an operating voltage of 10 $\mathrm{keV}$. A maximum beam current at the sample position of 10 nA was achieved by removing the objective aperture of the microscope. Total exposure times were varied from 10 to 1200 s. Magnetic images were taken with beam currents of $<0.3 \mathrm{nA}$ unless stated otherwise. All experiments were performed at a pressure of $1 \times 10^{-10}$ mbar.

The principle of local magnetic modification is illustrated in Fig. 1. An area measuring $18 \mu \mathrm{m} \times 18 \mu \mathrm{m}$ was scanned by the electron beam. The irradiation in the square corresponds to a dose of $2.4 \times 10^{10}$ electrons $/ \mu \mathrm{m}^{2}$. A magnetic image subsequently acquired at smaller magnification shows both the electron-beam-treated area and its surroundings. Within the square, the magnetization direction has switched completely from in-plane to out-of-plane as shown by the uniform gray level in Fig. 1(a) and the black/white contrast in Fig. 1(b). The square decays into a demagnetized state of up/down domains having typical sizes of several micrometers. The untreated film, on the other hand, consists of smaller, in-plane magnetized domains.

Figure 1 contains the essential aspect of the electronbeam-induced modifications reported in this paper: Magnetic anisotropy can be enhanced such that the magnetization can change its direction by large angles, up to a complete reorientation from parallel to perpendicular to the surface. Moreover, domain sizes can enlarge upon electron bombardment without an external magnetic field applied.

From the change in magnetization direction upon electron bombardment the change in anisotropy can be estimated by the standard phenomenological model. ${ }^{1}$ The free energy of the system is $E=\left(K_{1}-1 / 2 \mu_{0} M_{\mathrm{s}}^{2}+K_{\mathrm{s}} / d\right) \sin ^{2} \phi$ $+K_{2} \sin ^{4} \phi$, where $K_{1}$ and $K_{2}$ are the first- and second-order
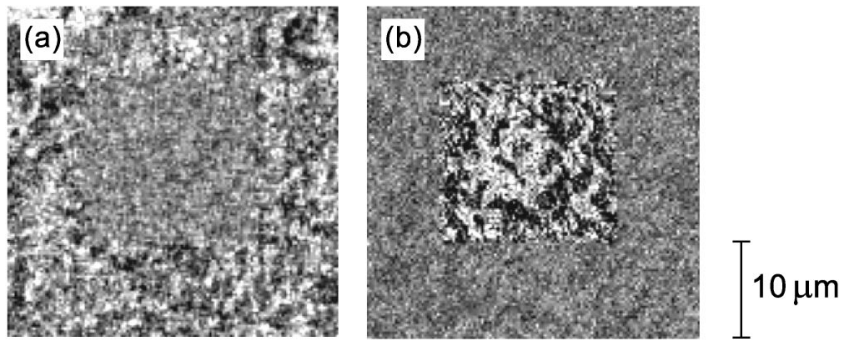

FIG. 1. Magnetic-domain images taken by spin-SEM after electron bombardment of a centered square for $1200 \mathrm{~s}$; (a) in-plane, and (b) out-of-plane magnetization component. The centered region has fully changed from being magnetized parallel to being magnetized perpendicular to the surface. Image size $35 \mu \mathrm{m} \times 35 \mu \mathrm{m}$. 


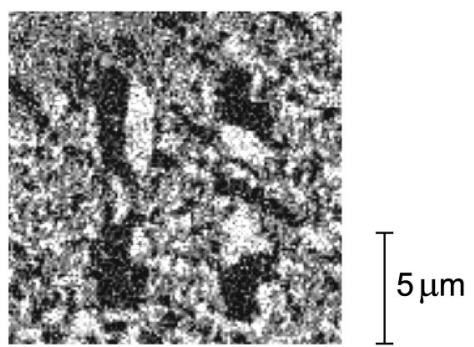

FIG. 2. Perpendicular magnetic-domain image after scanning four line segments arranged in a rectangular pattern of $3 \mu \mathrm{m}$ length from top to bottom for $60 \mathrm{~s}$. Note the induced domain size increase in an extended region. Image size $15 \mu \mathrm{m} \times 15 \mu \mathrm{m}$.

bulk anisotropy constants of Co, $K_{\mathrm{s}}$ the surface anisotropy constant, $M_{\mathrm{s}}$ the saturation magnetization, and $d$ the film thickness. In order to determine the angle $\phi$ between magnetization and surface normal we have grown a film with an initially canted magnetization direction of $60^{\circ}$ from the normal. Upon electron bombardment with a dose of $\approx 1$ $\times 10^{10}$ electrons $/ \mu \mathrm{m}^{2}$, a complete rotation toward the perpendicular direction is obtained. Assuming constant values for the parameters related to bulk properties, ${ }^{11}$ we deduce that the surface anisotropy has increased by at least $30 \%$, from $K_{\mathrm{s}} \approx 0.6$ to $0.8 \mathrm{~J} / \mathrm{m}^{2}$.

One might expect that an increase of the anisotropy beyond the minimum value necessary to stabilize complete perpendicular magnetization would not lead to a detectable change in the magnetic images because the magnetization direction is fully perpendicular already. This is not the case, however, as evidenced in Fig. 2. Here four vertical line segments, each $3 \mu \mathrm{m}$ long, arranged in a rectangular pattern were written into the Co film. The result of a local electronbeam bombardment with a dose of $2.5 \times 10^{11}$ electrons $/ \mu \mathrm{m}^{2}$ is an increase in domain size from 0.3 to $0.5 \mu \mathrm{m}$ in the as-grown film to $>2 \mu \mathrm{m}$. Both theoretical ${ }^{12}$ and experimental ${ }^{2}$ investigations have shown that the domain size of fully perpendicularly magnetized films in thermal equilibrium depends on anisotropy. We thus infer that the increased mean domain size upon electron-beam treatment is the result of a further increase of perpendicular anisotropy, and that the pinning strength of domain walls is reduced to such an extent that domain walls can propagate and actually reach equilibrium. Correspondingly, the modified areas decay into domains to maintain locally a demagnetized state.

Electron bombardment is thus one way to modify magnetic properties of an ultrathin Co film on a scale of $1 \mu \mathrm{m}$. Electron beams have much smaller diameters, but we were not successful in changing anisotropy on a submicrometer scale. For example, the modified regions of Fig. 2 were induced by a line scan of the intense electron beam: A beam radius of $100 \mathrm{~nm}$ spreads into a magnetically altered width of $\approx 2 \mu \mathrm{m}$. This is considerably larger than the range of secondary electrons in Co at $10 \mathrm{keV}$ primary energy, which is $0.3 \mu \mathrm{m} .{ }^{13}$

What is the origin of the anisotropy increase upon electron bombardment? An increase of surface anisotropy in the $\mathrm{Co} / \mathrm{Pt}$ system has been attributed to reduced interface roughness ${ }^{14}$ or, alternatively, to the formation of an alloy phase. ${ }^{15}$ Alloying at the interface has been reported to occur for temperatures above $350 \mathrm{~K}{ }^{16}$ Structurally more perfect Downloaded 31 Jan 2011 to 132.206 .203 .20 . Redistribution subject to AIP license or copyright; see http://apl.aip.org/about/rights_and_permissions

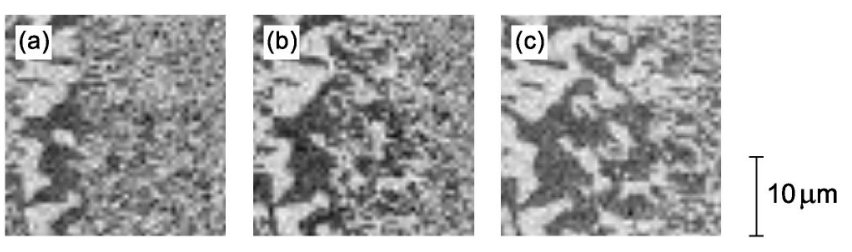

FIG. 3. Sequence of perpendicular magnetic domain images taken after scanning the rectangle on the left for $90 \mathrm{~s}$ with a beam current of $10 \mathrm{nA}$. Images (a) to (c) show repeated images taken with a reduced beam current of $1 \mathrm{nA}$. The perpendicularly magnetized phase evolves from the phase nucleated with increased beam current and finally fills the entire scanned area. The original film was magnetized in-plane. Image size $28 \mu \mathrm{m} \times 28 \mu \mathrm{m}$.

films exhibit larger surface anisotropies. We find that the $\mathrm{Pt}$ step density is directly correlated with the magnitude of the surface anisotropy, and that the surface anisotropy is reduced by $\mathrm{Ne}^{+}$bombardment. ${ }^{9}$ Hence, we can rule out that the creation of localized defects is the cause of anisotropy increase. Instead we think that electron irradiation helps the system evolve toward equilibrium, in contrast to the ionbombardment experiments in Ref. 7.

Highly intense electron beams are routinely used to anneal materials. ${ }^{17}$ However, beam powers similar to those used in the present study are expected to lead to a temperature increase of the order of only $0.03 \mathrm{~K} .^{13,18}$ Thus the anisotropy change is not induced by thermal annealing. Similarly we can also rule out interface alloying as a possible mechanism.

Electron bombardment has been shown to increase exchange coupling across $\mathrm{Fe} / \mathrm{Cr} / \mathrm{Fe}$ films. ${ }^{19}$ The results were interpreted in terms of a smoothening of the $\mathrm{Fe} / \mathrm{Cr}$ interface. These experiments were also characterized by a longdistance influence of the electron beam, extending even to millimeters rather than to micrometers as in our case. From their observations of beam voltage and beam-power dependencies, the authors of Ref. 19 concluded that a local twoelectron process from a core hole must be involved. This process transfers part of the relaxation energy to the lattice either by electron-phonon interaction or by creating a nucleus of a new structural phase.

Based on our experimental observations we conjecture that irradiation-induced nucleation of a new structural phase is the likely mechanism at work also in our Co/Pt film. Such a phase change would manifest itself in a corresponding change of the work function, which indeed is observed. The yield of secondary electrons is reduced by $2 \%$ in the entire magnetically modified area. ${ }^{20} \mathrm{We}$ found that it is easier to expand an already modified area than to create a new one, see Fig. 3. In this series of images, the rectangular region on the left was treated by an intense electron beam $(10 \mathrm{nA})$ until the magnetization direction pointed along the surface normal. Then, with a reduced beam current of $1 \mathrm{nA}$, the entire image was scanned repeatedly. The sequence of images shows that the perpendicularly magnetized phase evolves exclusively from the already existing perpendicular region. No new nucleation sites are created at this reduced beam current. This agrees with the argument that nucleation of a new phase requires more energy than phase expansion.

Whether this new phase is a modification of the Co film itself or of its interface is not clear to us. Both a reduced defect density within the film and a smoothed or intermixed 
interface are compatible with the observed work-function change. We think that the substrate plays a minor role in this process. Structural modifications require more activation energy in the bulk than at the interface or in the film because the number of intrinsic defects is much lower in the substrate.

In conclusion, we have modified magnetic anisotropies in $\mathrm{Co} / \mathrm{Pt}(111)$ films by electron bombardment on a micrometer scale. The changes in the perpendicular anisotropy are large enough to force the magnetization from a direction parallel to the surface to a fully perpendicular orientation. In films with perpendicular magnetization, magnetic domains can be expanded without applying an external magnetic field.

The authors thank O. Züger for the experiments testing the possibility of thermal annealing with an electron beam and for supplying the Ga single crystal.

${ }^{1}$ P. Beauvillain, A. Bounouh, C. Chappert, R. Mégy, S. Ould-Mahfoud, J. P. Renard, and P. Veillet, J. Appl. Phys. 76, 6078 (1994).

${ }^{2}$ M. Speckmann, H. P. Oepen, and H. Ibach, Phys. Rev. Lett. 75, 2035 (1995).

${ }^{3}$ W. Weber, C. H. Back, U. Ramsperger, A. Vaterlaus, and R. Allenspach, Phys. Rev. B 52, R14400 (1995).

${ }^{4}$ S. Hope, E. Gu, B. Choi, and J. A. C. Bland, Phys. Rev. Lett. 80, 1750 (1998).
${ }^{5}$ R. J. Pollard, M. J. Wilson, and P. J. Grundy, J. Appl. Phys. 76, 6090 (1994)

${ }^{6}$ W. Weber, C. H. Back, A. Bischof, D. Pescia, and R. Allenspach, Nature (London) 374, 788 (1995).

${ }^{7}$ C. Chappert, H. Bernas, J. Ferré, V. Kottler, J. P. Jamet, Y. Chen, E. Cambril, F. Rousseaux, V. Mathet, and H. Launois, Science 280, 1919 (1998).

${ }^{8}$ P. Grütter and U. Dürig, Phys. Rev. B 49, 2021 (1994).

${ }^{9}$ R. Allenspach, U. Dürig, and P. Grütter (unpublished).

${ }^{10}$ R. Allenspach, J. Magn. Magn. Mater. 129, 160 (1994).

${ }^{11} M_{\mathrm{s}}=1440 \mathrm{kA} / \mathrm{m}, K_{1}=412 \mathrm{~kJ} / \mathrm{m}^{3}, K_{2}=143 \mathrm{~kJ} / \mathrm{m}^{3}$.

${ }^{12}$ B. Kaplan and G. A. Gehring, J. Magn. Magn. Mater. 128, 111 (1993).

${ }^{13}$ L. Reimer and G. Pfefferkorn, Raster-Elektronenmikroskopie (Springer, Berlin, 1973).

${ }^{14}$ C.-H. Chang and M. H. Kryder, J. Appl. Phys. 75, 6864 (1994).

${ }^{15}$ D. Weller, H. Brändle, and C. Chappert, J. Magn. Magn. Mater. 121, 461 (1993).

${ }^{16}$ J. S. Tsay and C. S. Shern, J. Appl. Phys. 80, 3777 (1996).

${ }^{17}$ M. Cervera, J. Martinez, J. Garrido, and J. Piqueras, Appl. Phys. A: Mater. Sci. Process. 62, 451 (1996).

${ }^{18} \mathrm{We}$ explicitly tested the possibility of thermal annealing by trying to melt a Ga single crystal kept $0.2 \mathrm{~K}$ below the melting temperature. We were unable to melt it with the same beam parameters as used for magnetic modifications.

${ }^{19}$ S. O. Demokritov, J. A. Wolf, and P. Grünberg, Appl. Phys. Lett. 63, 2147 (1993).

${ }^{20}$ Film contamination by cracked organic molecules as observed with standard SEM (Ref. 13) is ruled out because of our ultrahigh vacuum environment and from the absence of contamination peaks in Auger electron spectroscopy. 